\title{
Spatial effectiveness of the mask: \\ Lateral inhibition in visual backward masking'
}

\author{
DEAN G. PURCELL, ${ }^{2}$ ALAN L. STEWART, AND WILLIAM N. DEMBER \\ UNIVERSITY OF CINCINNATI
}

\begin{abstract}
Introducing a figure into a masking flash results in visual backward masking under conditions where a homogeneous masking flash does not suppress target detection. It is possible to analyze the spatial effects of such a masking figure in terms of lateral inhibition. It is hypothesized that incorporating a figure into the masking flash changes the inhibitory pattern the mask produces in the visual system. The interaction between the firing pattern produced by the mask and the residual inhibition from the preceding target presentation results in a phenomenal representation different from that produced by either the target or the mask alone.
\end{abstract}

A briefly presented black target on a white surround may be suppressed by a subsequent flash of white light (Thompson, 1966). Targets which are not susceptible to the "backward masking" effect of a homogeneous flash of light may be suppressed if the masking stimulus contains a figure with its contour near the target contour (Eriksen \& Collins, 1965). We have previously proposed (Purcell \& Dember, 1968) that masking by homogeneous flashes might be mediated by the mechanism of lateral inhibition as found in the eye of Limulus (Ratliff, 1965). In this paper we elaborate that proposal so as to incorporate the spatial gradient of effectiveness of the masking figure.

The following assumptions are consistent with steady state data from the Limulus. (1) The firing of an aggregate of neural cells in the visual system has the effect of inhibiting or lowering the firing rate of those and adjacent cells to present and subsequent stimulation. (2) The amount of inhibition generated by a given flash of light is an increasing function of the intensity of that flash. (3) Inhibition decays as a monotonic function of time since termination of stimulation. (4) The relative amount of inhibition on a given neuron, firing or nonfiring, is a positive function of the number and proximity of firing neurons surrounding it. Thus, the rate of firing generated by a given visual stimulus is not simply a function of the physical energy of that stimulus, but is susceptible to modulation by inhibition already present in the visual system as well as inhibition generated by that stimulus itself.

Application of the preceding model to the two-flash visual backward masking paradigm requires one additional assumption. (5) The phenomenal brightness of a given visual stimulus or of a train of intermittent stimuli results from the integration of firing to that stimulus presentation within some critical time period.

From the preceding assumptions an explanation of visual backward masking can be developed. For purposes of exposition, let us consider two aggregates of neurons in the visual system: Group 1, those neurons upon which a black target impinges and Group 2, those neurons upon which the target's bright surround impinges. With the presentation of the target flash Group 2 fires and generates inhibition in its area. Group 1 fires relatively little or not at all and generates relatively little or no inhibition. As a consequence of this differential in inhibition Group 1 and 2 are differentially susceptible to excitation by a subsequent homogeneous masking flash. Thus, with the presentation of the masking flash, Group 1, being uninhibited, is transformed from an area of relatively low rates of firing to an area of relatively high rates of firing. Group 2 is transformed from an area of relatively high rates of firing to an area of relatively low rates of firing.

Since the perceived brightness of the area served by each group of neurons is a function of the temporal integration of their respective firing rates to the target and masking flashes, three phenomenal outcomes of target and mask presentation are possible (Purcell \& Dember, 1968). Each outcome is dependent upon the differential in inhibition between Groups 1 and 2 generated by the target flash. If the differential is sufficient, then Group 1's firing to the masking flash will be much greater than Group 2's and integration will result in a target figure which is phenomenally brighter than its surround (what we have called "brightness reversal"). With relatively less of a differential, integration will result in a target figure which is phenomenally the same brightness as its surround and not discriminable from it. With even a smaller differential, integration will result in a target figure with a phenomenal appearance ranging from grey to black. The first two outcomes are such as to produce a mismatch between the phenomenal appearance of the target and a S's criterion of response in a detection task. Hence, the target is effectively masked.

In the case of the third outcome, if masking is to obtain, then the firing rate of Group 1 must be increased relative to that of Group 2 . One way to accomplish this is to increase inhibition in Group 2 by increasing the luminance of the target surround, but let us consider another method. According to assumption four, introducing a black area (i.e., a masking figure) into the masking flash will serve to protect neurons adjacent to and surrounding the masking figure from receiving as much lateral inhibition as they would if the masking field was homogeneous. Consequently, those neurons adjacent to the masking figure can fire at a higher rate than when stimulated by a homogeneous masking flash. A masking figure will increase the rate of firing of Group 1 neurons more than it will that of Group 2, simply because the target presentation has left Group 2 inhibited more than Group 1. If the increase in firing rate of Group 1 relative to Group 2 is great enough, then masking will be accomplished.

Within the limits of parameters where a homogeneous masking flash is not an effective mask, the following relationships are postulated. As the ISI is decreased, or the luminance of the target presentation is increased, it is expected that the differential in inhibition present for a homogeneous masking flash to act upon will increase. The greater this differential the greater will be the spatial effectiveness of a masking figure present in the masking flash. This is because small increases in firing rate on neurons distal to the masking figure will be sufficient to yield the increase in firing of Group 1 over Group 2 necessary to accomplish masking. More specifically, it is hypothesized that the effects of target-field luminance and ISI will be manifest in the spatial extent over which the masking figure has impact.

\section{Procedure}

Testing the hypotheses necessitated the use of stimuli which differed in shape from those generally encountered in masking experiments. The mask consisted of a black bar $(28.8 \mathrm{~min} \times 91$ min) which extended from the left side to the middle of a 182 min square white masking field. The target consisted of a black bar (28.8 min in width, length variable from 0 to $67.2 \mathrm{~min}$ ) which could be extended from the middle toward the right side of a white (182-min square) target field. Simultaneous exposure of target and masking fields, as the target was extended, gave the phenomenal appearance of a black bar growing in length to the right until almost horizontally bisecting the field (see Fig. la). Presentation of stimuli was by a tachistoscope (Scientific Prototype GB) 


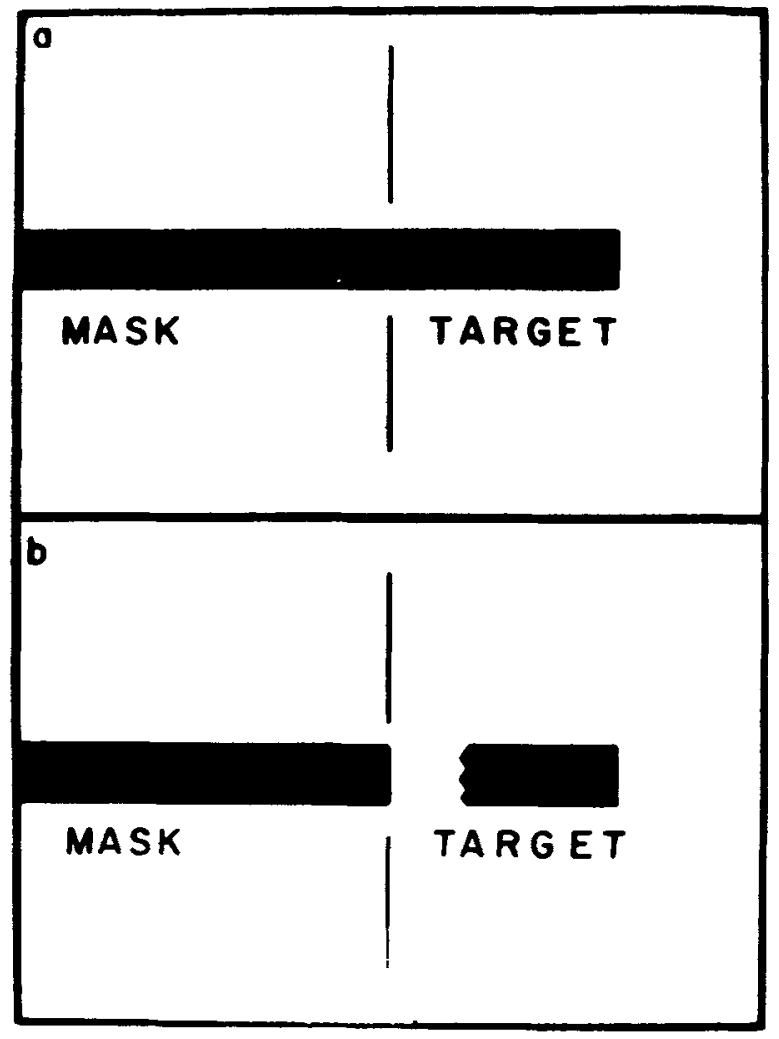

Fig. 1. (a) The phenomenal appearance of the target and mask when exposed simultaneously. (b) The phenomenal appearance of the target when the target presentation is followed by the mask.

which effectively superimposed the target and masking fields and controlled stimulus onset and of fset asychrony.

Pilot data gathered on seven naive Ss demonstrated that when a fully extended target was followed by the bar mask. those areas of the target closest to the mask could not be seen. and were effectively masked. while portions of the target further to the right of the mask were clearly seen (see Fig. 1b).

The following parametric study was run to systematically investigate the extensive characteristics of the area of effective masking in accordance with changes in target field luminance and 1SI. The target field was exposed for $5 \mathrm{msec}$, lts luminance was varied from $30 \mathrm{ft}-\mathrm{L}$ to $60 \mathrm{ft}-\mathrm{L}$ in $10 \mathrm{ft}-\mathrm{L}$ steps. The contrast ratio between the target surround and the target bar proper remained constant at .12. The unilluminated ISI was varied from 10 to 50 msec in $10 \mathrm{msec}$ steps. The luminance of the masking field was 40 $\mathrm{ft}-\mathrm{L}$ (field-to-mask ratio, .12) and was exposed for $75 \mathrm{msec}$. The durations of target, ISI, and mask were chosen on the basis of preliminary investigations to assure reliable masking effects. Similarly, the luminance of the target field was chosen to assure that the target would not be masked by a homogeneous masking flash. Twenty trials, under each masking condition, were run on each of five trained, paid Ss. After a 5-min dark adaptation period. Ss were allowed to initiate each trial after a "ready" signal from the E. During the 5 to $10 \mathrm{sec}$ dark intertrial interval Ss fixated on the center of the presentation field as defined by the intersection of imaginary vertical and horizontal lines going from dim red peripheral points contained in that field. All viewing was with the right eye.

Estimates of the area of effective masking were obtained by extending the target bar from the middle (i.e., out from the masking bar) toward the right of thi presutation field. Ss were instructed to respond "yes" on thes: trials when a portion of the black target bar was seen extendin!: beyond the milline of the masking field, and "no" when it "as not. The "up and down" sensitivity method (Dixon \& Vissey. 1951) was employed. Essentially this is a modified ntethod of limits in which two independent ascending and descending series, 10 trials each, were randomly interspersed with one another. One series began descending with the target extended far enough to elicit a "yes" response. The target was then retracted toward the nlask $4.8 \mathrm{ft}$ on each trial in that series until in "no" response was made. Then the direction of this series was reversed until a "yes" response was made, and so on until that serits was exhausted. The other series was run in the same manner except that it was begun as an ascending series. with the target not extended far enough from the mask to elicit a "yes" response. since trials from the two series were randomly interspersed. the $S$ could not ascertain on any given trial whether he was on the ascending or descending series. With the above method. the mean point of target extension at which the target was detected was obtained for all conditions. The greater the extension of the target bar necessary for detection, the greater the spatial effectiveness of the masking bar.

\section{RESULTS AND DISCUSSION}

Table 1 gives the amount of target extension (in minutes of visual angle) necessary for detection averaged over the five Ss. Inspection of this table reveals that the effect of target field luminunce on target extension is negligible. while ISI has a strong and consistent effect on target extension $(F=14.65, \mathrm{df}=4 / 16$, $\mathrm{p}<.001)$. The shorter the ISI. the farther the target must be extended from the mask to be seen.

The finding that the spatial effectiveness of the mask increased as ISI decreased directly supports the findings of Levine, Didner. and Tobenkin (1907) and is analogous to findings demonstrating that target detectability decreases as ISI is decreased (Raab. 1963). Although increases in target luminance have no systematic effect in this study, the authors huve recently demonstrated reduced target defectability as a function of increased target-field luminance (Purcell. Stewart. \& Dember, in preparation). The present study as well as the others cited give direct support to the application of the concept of lateral inhibition. as stated in this paper, to the explanation of visual backward masking.

Those values in Table 1 that are underscored with asterisks represent parameters at which a maximum extension of the target bar was undetected. The number of asterisks represents the number of Ss failing to detect a maximum extension. An arbitrary score of $67.2 \mathrm{~min}$ was assigned in these cases for purposes of the analysis.

The parameters where maximum target extensions were undetected are those which we believe are most conducive to

Table 1

Mean Target Extension to Obtain Detection as a Function of Interstimulus Interval and Luminance Level

\begin{tabular}{ccccc}
\hline Interstimulus Interval (msec) & \multicolumn{4}{c}{ Target Luminance (ft-L) } \\
\cline { 2 - 5 } & 30 & 40 & 50 & 60 \\
\hline 10 & $59.63^{\prime}$ & $61.31^{\prime}$ & $67.20^{\prime}$ & $67.20^{\prime}$ \\
20 & $42.96^{\prime}$ & $41.04^{\prime}$ & $40.14^{\prime}$ & $42.40^{\prime}$ \\
30 & $29.37^{\prime}$ & $26.65^{\prime}$ & $35.78^{\prime}$ & $39.47^{\prime}$ \\
40 & $18.67^{\prime}$ & $23.81^{\prime}$ & $28.85^{\prime}$ & $22.90^{\prime}$ \\
50 & $12.76^{\prime}$ & $11.52^{\prime}$ & $14.94^{\prime}$ & $15.88^{\prime}$ \\
\hline
\end{tabular}

Asterisks denote the parameters at which a full extension of the target was und tected. Number of asterisks correspond to the number of Ss for which this occurred. 
obtaining effective masking without the influence of a masking figure. To check this, three Ss, run in the initial experiment, were later run under those parameters at which each individual could not detect a maximum extension of the tanget bar. The masking bar was removed from the masking flash, and the Ss were asked to report on the phenomenal appearance of the target bar. When target and masking flash were presented, the Ss reported that the target bar was either not evident or appeared as a bright (i.e., "reversed") bar on a darker surround. This indicates that at the underscored parameters in Table 1 the masking bar was contributing little, if at all, to effective masking.

\section{REFERENCES}

DIXON, W., \& MASSEY, F. Introduction to statistical analysis. New York: Toronto, London: McGraw-Hill, 1951.

ERIKSEN, C. W., \& COLLINS, J. F. A reinterpretation of one form of backward and forward masking in visual perception. Joumal of Experimental Psychology, 1965, 70, 343-351.

LEVINE, R., DIDNER, R., \& TOBENKIN, N. Backward masking as a function of interstimulus distance. Psychonomic Science, 1967, 9, 185-186.
PURCELL, D. G., \& DEMBER, W. N. The relation of phenomenal brightness reversal and re-reversal to backward masking and recovery. Perception a Psychophysics, 1968, 3, 290-292.

PURCELL, D. G., STEWART, A. L., \& DEMBER, W. N. Backward masking: Facilitation through increased target-field luminance and duration. In preparation.

RAAB, D. H. Backward masking. Psychological Bulletin, 1963, 60, 118-119. RATLIFF, F. Mach Bands: Quantitative studies on neural networks in the retina. San Francisco: Holden Day, 1965.

IHOMPSON, J. H. What happens to the stimulus in backward masking? Joumal of Experimental Psychology, 1966, 71, 580-586.

\section{NOTES}

1. Supported by Grant No. NB-07622-02 to W. N. D. from the National Institutes of Health.

2. Now at the University of Toron to, Toronto, Canada.

(Accepted for publication August 16, 1968.) 